Correspondence

Maciej Ugorski

maciej.ugorski@up.wroc.pl

Received 22 February 2010

Revised 16 March 2010

Accepted 16 March 2010

\section{The high-adhesive properties of the FimH adhesin of Salmonella enterica serovar Enteritidis are determined by a single F118S substitution}

\author{
Krzysztof Grzymajło, ${ }^{1}$ Marta Kuźmińska-Bajor, ${ }^{1}$ Jakub Jaworski, ${ }^{1}$ \\ Piotr Dobryszycki ${ }^{2}$ and Maciej Ugorski ${ }^{1,3}$
}

\author{
${ }^{1}$ Department of Biochemistry, Pharmacology and Toxicology, Faculty of Veterinary Medicine, \\ University of Environmental and Life Sciences, C. Norwida 31, 50-375 Wrocław, Poland \\ ${ }^{2}$ Division of Biochemistry, Institute of Organic Chemistry, Biochemistry and Biotechnology, Wrocław \\ University of Technology, Wybrzeże Wyspiańskiego 27, 50-370 Wrocław, Poland \\ ${ }^{3}$ Department of Glycobiology and Cell Interactions, Ludwik Hirszfeld Institute of Immunology and \\ Experimental Therapy, Polish Academy of Sciences, R. Weigla 12, 53-114 Wrocław, Poland
}

\begin{abstract}
The binding properties of low- and high-adhesive forms of FimH adhesins from Salmonella enterica serovars Enteritidis and Typhimurium ( $S$. Enteritidis and S. Typhimurium) were studied using chimeric proteins containing an additional peptide that represents an $\mathrm{N}$-terminal extension of the FimF protein. This modification, by taking advantage of a donor strand exchange mechanism, closes the hydrophobic groove in the fimbrial domain of the FimH adhesin. Such selfcomplemented adhesins ( $\mathrm{scFimH}$ ) did not form aggregates and were more stable (resistant to proteolytic cleavage) than native $\mathrm{FimH}$. High-adhesive variants of scFimH proteins, with alanine at position 61 and serine at position 118, were obtained by site-directed mutagenesis of fimH genes from low-adhesive variants of $S$. Enteritidis and $S$. Typhimurium, with glycine at position 61 and phenylalanine at position 118. Direct kinetic analysis using surface plasmon resonance (SPR) and glycoproteins carrying high-mannose carbohydrate chains (RNase $B$, horseradish peroxidase and mannan-BSA) revealed the existence of high- and low-adhesive allelic variants, not only in $S$. Typhimurium but also in $S$. Enteritidis. Using two additional mutants of low-adhesive FimH protein from S. Enteritidis (Gly61Ala and Phe118Ser), SPR analysis pointed to Ser118 as the major determinant of the high-adhesive phenotype of type 1 fimbriae from $S$. Enteritidis. These studies demonstrated for the first time that the functional differences observed with whole fimbriated bacteria could be reproduced at the level of purified adhesin. They strongly suggest that the adhesive properties of type 1 fimbriae are determined only by structural differences in the FimH proteins and are not influenced by the fimbrial shaft on which the adhesin is located.
\end{abstract}

\section{INTRODUCTION}

Fimbriae are proteinaceous filamentous structures present on the surface of many members of the Enterobacteriaceae (Duguid et al., 1966; Thorns, 1995). An increasing amount of evidence suggests that type 1 fimbriae play an important role in attachment to and colonization of the gut mucosa by different serovars of Salmonella enterica, including Salmonella Enteritidis and Salmonella Typhimurium (De Buck et al., 2003; Dibb-Fuller et al., 1999; Dibb-Fuller \&

Abbreviations: $C D$, circular dichroism; HRP, horseradish peroxidase; SPR, surface plasmon resonance.

Two supplementary figures, showing HPLC elution profiles of scFimH adhesins and SDS-PAGE of mutated EscFimH/L proteins, are available with the online version of this paper.
Woodward, 2000; Ewen et al., 1997; Naughton et al., 2001). In addition, it has recently been shown that internalization of $S$. Typhimurium by murine dendritic cells can be initiated by a type 1 fimbriae-dependent mechanism (Guo et al., 2007). However, their precise role in the pathogenesis of Salmonella is not clear (Allen-Vercoe \& Woodward, 1999; Allen-Vercoe et al., 1999; Rajashekara et al., 2000).

Type 1 fimbriae are rod-like surface structures $7 \mathrm{~nm}$ thick and up to $3 \mu \mathrm{m}$ in length, composed primarily of protein subunits called FimA (Jones et al., 1995). The adhesive properties of type 1 fimbriae depend on a lectin-like FimH adhesin located on the tip of the fimbrial shaft (Krogfelt et al., 1990). FimH binds to oligomannosidic structures present on many eukaryotic membrane-bound and secreted glycoproteins (Firon et al., 1983, 1984). 
Based on the early studies, it was proposed that enterobacterial type 1 fimbriae are structurally related (Abraham et al., 1988); however, recent data have shown significant heterogeneity among FimH adhesins from different genera and even within the same species or serovar with respect to binding properties for mannosylated ligands (Sokurenko et al., 1994, 1995). On the serovar level we recently showed that inactive FimH adhesins from Salmonella Gallinarum biovars Gallinarum and Pullorum are characterized by the presence of isoleucine at position 78 instead of the threonine found in the active FimH adhesins of $S$. Typhimurium and $S$. Enteritidis (Kisiela et al., 2005a, 2006). At the strain level, Boddicker et al. (2002) have found that two strains of $S$. Typhimurium, which express allelic variants of FimH protein differing by two amino acid residues (Gly61Ala and Phe118Ser), adhere weakly and strongly to human HEp-2 cells.

Sequencing of the fim $H$ genes from different avian and human S. Enteritidis isolates (Kisiela et al., 2005b) shows that all the analysed strains represent the low-adhesive variant of $S$. Typhimurium described by Boddicker et al. (2002). The functional characterization of low-adhesive variants of FimH proteins from $S$. Enteritidis and $S$. Typhimurium reveals that FimH adhesins bind equally to human colon carcinoma and bladder cancer cells (Kisiela et al., 2006). In contrast, the high-adhesive variant of $S$. Typhimurium FimH adhesin binds to mouse enterocytes, but not to mouse uroepithelial cells, as shown by Thankavel et al. (1999). To explain these discrepancies we hypothesized that only the high-adhesive variants of FimH proteins are able to discriminate between different eukaryotic cells, in contrast to the low-adhesive forms of FimH proteins (Kisiela et al., 2006). As in our study the purified FimH proteins were used, the other possible explanation is that the binding specificity of FimH adhesins is modulated by the fimbrial shaft on which this adhesin is presented (Duncan et al., 2005). In the present study we addressed this problem by constructing low- and highadhesive variants of FimH proteins from $S$. Enteritidis and $S$. Typhimurium, using site-directed mutagenesis. Their binding properties were studied at the molecular level using purified recombinant protein.

\section{METHODS}

Construction of EextfimH.LA and TextfimH.LA vectors containing variants of the fimH gene with an $\mathrm{N}$-terminal extension of the fimF gene and myc epitope- and His-tags. ssDNA oligonucleotides with sequences corresponding to the first 17 amino acids of the $S$. Enteritidis FimF protein [N-terminal extension containing additional sequences corresponding to a four amino acid linker (Glu-Lys-Asp-Asn) (Barnhart et al., 2000) and XbaI restriction sites at the $5^{\prime}$ and $3^{\prime}$ termini (5'-CTAGGTCCACCACGTTACCGCGCAGTTCAATATTCACTTCGCCCAGAGATGAGTTCTGTTTATTAT $\left.-3^{\prime}\right)$ ] were obtained from the Institute of Biochemistry and Biophysics, Polish Academy of Sciences, Warsaw, Poland. They were hybridized to make dsDNA and ligated to the corresponding restriction sites in the vectors T.fimH/pTrcHis2b or E.fimH/
pTrcHis2b containing fimH genes from $S$. Typhimurium and $S$. Enteritidis, respectively (Kisiela et al., 2005a). The new constructs were used as templates to amplify, by PCR, fimH-N-terminal fimF extension fusion genes containing a myc epitope-tag with primers $5^{\prime}$ extfimH/myc (5'-GCATCCATGGCGACGGTTTGC- $\left.3^{\prime}\right)$ and $3^{\prime}$ extfimH/myc ( $5^{\prime}$-CAGAAGAGGATCTGAATAAGCTTCGC-3'). The PCR primers contained additional sequences corresponding to the $\mathrm{NcoI}$ restriction site in the primer $5^{\prime}$-extfimH/myc and to HindIII in $3^{\prime}$-extfimH/myc. The gene constructs were amplified as follows: hot start $\left(94{ }^{\circ} \mathrm{C}\right.$ for $\left.5 \mathrm{~min}\right)$, then 24 cycles of denaturation $\left(94{ }^{\circ} \mathrm{C}\right.$ for $50 \mathrm{~s})$, annealing $\left(59^{\circ} \mathrm{C}\right.$ for $\left.50 \mathrm{~s}\right)$, elongation $\left(72{ }^{\circ} \mathrm{C}\right.$ for $\left.90 \mathrm{~s}\right)$ and terminal elongation at $72{ }^{\circ} \mathrm{C}$ for $5 \mathrm{~min}$. The PCR amplicons were digested with the respective enzymes and ligated to the corresponding sites of plasmid pET-22b (Novagen). The resulting vectors contained the low-adhesive variants of fimH genes from $S$. Enteritidis and $S$. Typhimurium linked to the N-terminal extension of the fimF gene from $S$. Enteritidis, a myc-tag and a His-tag, and were named EextfimH.LA and TextfimH.LA, respectively.

Site-directed mutagenesis of fimH genes. To construct highadhesive variants of the fimH genes from $S$. Enteritidis and $S$. Typhimurium, mutagenesis of their respective low-adhesive variants of the fim $H$ genes linked to the $\mathrm{N}$-terminal extension of the fim $F$ gene from $S$. Enteritidis was performed using a PCR-based method. The mutations of interest were generated by two separate PCRs using two pairs of primers. PCR I was carried with the common $5^{\prime}$ primer (fimH-1, 5'-CGCGGATCCAATGAAAATATACTCAGC-3') and 3' primers with the desired Gly61Ala (primer fimH-2, 5'-GCTGGGTTCGTCTAAAGAGGCCGTCG-3') or Phe118Ser (primer fimH-3, 5' CGCCTATTCTATGCGGTCGCTGTGATGC-3') mutations and the plasmids E.fimH/pTrcHis2b and T.fimH/pTrcHis2b as templates (Kisiela et al., 2005a). PCR II was performed with the common $3^{\prime}$ primer (fimH-4, 5'-GCGTCTAGAGCATCATAATCGACTCG-3') and $5^{\prime}$ primers with the desired Gly61Ala (fimH-5, 5'-GCTGCCGGAGAAATCTGCTTGGGTCG-3') and Phel18Ser (fimH-6, 5' CGATAGTGTCGCTGGCGTATCTTATCCGC-3') mutations. The first and second PCRs were performed as follows: (I) hot start $\left(2 \mathrm{~min}, 94{ }^{\circ} \mathrm{C}\right)$, then 20 cycles of denaturation $\left(50 \mathrm{~s}, 94{ }^{\circ} \mathrm{C}\right)$, annealing $\left(50 \mathrm{~s}, 57^{\circ} \mathrm{C}\right)$ and elongation $\left(1 \mathrm{~min}, 72^{\circ} \mathrm{C}\right)$ for the Gly61Ala mutation; and (II) hot start $\left(2 \mathrm{~min}, 94^{\circ} \mathrm{C}\right)$, then 20 cycles of denaturation $\left(50 \mathrm{~s}, 94{ }^{\circ} \mathrm{C}\right)$, annealing $\left(50 \mathrm{~s}, 55^{\circ} \mathrm{C}\right)$ and elongation $\left(72{ }^{\circ} \mathrm{C}, 1 \mathrm{~min}\right)$ for the Phel18Ser mutation. The resulting DNA fragments were used as templates for a third PCR with primers fimH1 and fimH-4. The reaction was performed as follows: hot start $\left(2 \mathrm{~min}, 94{ }^{\circ} \mathrm{C}\right)$, then 20 cycles of denaturation $\left(1 \mathrm{~min}, 94{ }^{\circ} \mathrm{C}\right)$, annealing $\left(1 \mathrm{~min}, 52{ }^{\circ} \mathrm{C}\right)$ and elongation $\left(1 \mathrm{~min}, 72{ }^{\circ} \mathrm{C}\right)$. The mutated fim $H$ genes were cloned into plasmid pET-22b and the expression vectors containing the high-adhesive variants of the fimH genes from $S$. Enteritidis and $S$. Typhimurium were designated EextfimH.HA and TextfimH.HA, respectively.

Two additional mutants of the FimH protein were made using a method based on the QuikChange Site-Directed Mutagenesis kit (Stratagene). The Gly61Ala mutant was made by a single PCR using the primers 5'-GCCGGAAAAATCTGCTTGGGTCGGCG-3' and 5'CGCCGACCCAAGCAGATTTTCCGGCGC- $3^{\prime}$ and the EextfimH.LA vector as template. The Phe118Ser mutant was obtained by a single PCR using the primers 5'-CGCTGGCGTATCTTATCCGCCCCGTAAC-3' and 5' ${ }^{\prime}$-CGGGGCGGATAAGATACGCCAGGACAC- ${ }^{\prime}$ and the same vector as template. The mutants were amplified as follows: hot start $\left(2 \mathrm{~min}, 95{ }^{\circ} \mathrm{C}\right)$, then 14 cycles of denaturation $\left(45 \mathrm{~s}, 95{ }^{\circ} \mathrm{C}\right)$, annealing $\left(1 \mathrm{~min}, 55^{\circ} \mathrm{C}\right)$ and elongation $\left(6 \min 40 \mathrm{~s}, 68^{\circ} \mathrm{C}\right)$. The reaction mixture contained high-fidelity OptiTaq DNA polymerase (EURx, Gdańsk, Poland) and $D p n I$ restriction enzyme to remove the original template. The mutations introduced into the $\mathrm{fim} H$ gene were confirmed by DNA sequencing. As above, the mutated fim $H$ genes 
were cloned into plasmid pET-22b, and the resulting vectors were designated EextfimH.Gly61Ala and EextfimH.Phe118Ser.

Expression and purification of recombinant self-complemented FimH proteins (scFimHs). FimH proteins self-complemented with the N-terminal extension of the FimF protein (scFimH) and representing the high- and low-adhesive variants were named, respectively, EscFimH/H and EscFimH/L for $S$. Enteritidis, and TscFimH/H and TscFimH/L for S. Typhimurium. Escherichia coli BL2 1 cells (Novagen), transformed with the appropriate expression vector, were grown at $37{ }^{\circ} \mathrm{C}$ in $250 \mathrm{ml}$ Luria-Bertani (LB) medium containing $25 \mathrm{mM} \mathrm{MgCl}, 0.4 \%$ glycerol (v/v) and ampicillin $\left(100 \mu \mathrm{g} \mathrm{ml}^{-1}\right)$. When the $\mathrm{OD}_{600}$ of the growing culture reached 0.6 , IPTG (Fermentas) was added to a final concentration of $0.5 \mathrm{mM}$. The temperature was lowered to $26{ }^{\circ} \mathrm{C}$ and bacterial growth was continued for another $12 \mathrm{~h}$. Bacteria were centrifuged and lysed with $20 \mathrm{mM}$ Tris/HCl, $\mathrm{pH} 7.8$, containing $0.1 \mathrm{M} \mathrm{NaCl}$ and $8 \mathrm{M}$ urea. The presence of urea in lysed buffer increased the yield of the final purification by about 10 times. The recombinant proteins were purified using Ni-NTA affinity chromatography (Sigma-Aldrich), concentrated on Centricon 10 filters (Millipore) and quantified by a bicinchoninic acid (BCA) protein assay kit (Sigma).
Biotinylation of FimH proteins was performed as previously described (Kisiela et al., 2005a).

Synthesis of mannosylated BSA (mannan-BSA). Mannan-BSA was synthesized according to the method of Kanska et al. (2008). Briefly, mannan (Sigma) was oxidized by $0.5 \%$ sodium periodate in $0.1 \mathrm{M}$ acetate buffer, $\mathrm{pH}$ 5.4. The reaction was carried out for $1 \mathrm{~h}$ at $4{ }^{\circ} \mathrm{C}$ in the dark. Four milligrams of oxidized mannan was mixed with $12 \mathrm{mg}$ BSA (Sigma) $(1: 3, \mathrm{w} / \mathrm{w})$. The samples were frozen in an acetone/dry ice bath and lyophilized, then heated at $118 \pm 2{ }^{\circ} \mathrm{C}$ for $20 \mathrm{~min}$ and dissolved in Tris-buffered saline (TBS; $10 \mathrm{mM}$ Tris/ $\mathrm{HCl}$, $150 \mathrm{mM} \mathrm{NaCl}, \mathrm{pH}$ 7.4). The efficiency of mannan-BSA conjugation was confirmed by SDS-PAGE.

SDS-PAGE and Western blotting. The purified scFimH proteins were analysed in Coomassie Brilliant Blue-stained SDS-PAGE gels $(12 \%)$. They were identified by Western blotting using primary antimyc mAb 9E10 (ATCC, no. CRL-1729) and alkaline phosphataseconjugated goat anti-mouse immunoglobulins (Dako) or rabbit polyclonal antibodies directed against FimH (Kisiela et al., 2005b) and horseradish peroxidase (HRP)-conjugated goat anti-rabbit immunoglobulins (Sigma). The binding of the scFimH proteins to RNase B (Sigma), HRP (Sigma) and mannan-BSA was analysed by far-Western blotting. High-mannose glycoproteins, after SDS-PAGE

(a)

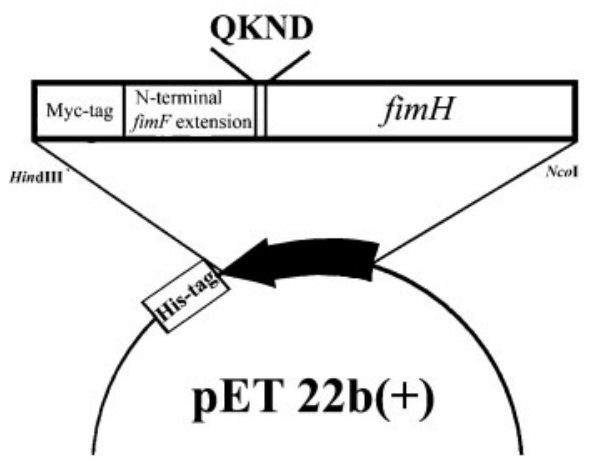

(b)

(i)

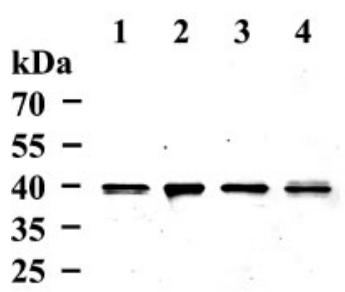

$25-$ (ii)

$\begin{array}{llll}1 & 2 & 3 & 4\end{array}$

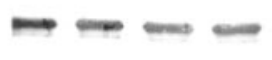

(iii)

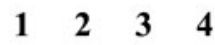

Fig. 1. (a) Schematic representation of fimH gene constructs with an $\mathrm{N}$-terminal extension of the fimF gene and a myc epitopetag. (b) (i) Coomassie blue-stained SDS-PAGE, and immunostaining with (ii) rabbit polyclonal anti-FimH antibodies and (iii) anti-myc mAb $9 \mathrm{E} 10$ of FimH proteins with an N-terminal extension of the FimF protein from S. Enteritidis (scFimH). Lanes 1 and 3 , low-adhesive variants of scFimH adhesins from $S$. Enteritidis and $S$. Typhimurium, respectively; lanes 2 and 4, high-adhesive variants of scFimH proteins from $S$. Enteritidis and $S$. Typhimurium, respectively. scFimH proteins $(10 \mu \mathrm{g})$, purified on Ni-NTA resin, were subjected to SDS-PAGE under reducing conditions in a $12 \%$ gel. The migration positions of protein standards (in $\mathrm{kDa}$ ) are indicated on the left. 
in a $10 \%$ gel, were transferred to nitrocellulose (Bio-Rad), blocked overnight with $5 \%$ fat-free dry milk in TBST (TBS supplemented with $0.2 \%$ Tween 20$)$ and incubated with scFimH proteins $(0.2 \mathrm{mg}$ $\mathrm{ml}^{-1}$ ) for $12 \mathrm{~h}$. Immunodetection was performed using anti-myc $\mathrm{mAb} 9 \mathrm{E} 10$ and alkaline phosphate-conjugated goat anti-mouse immunoglobulins.

HPLC. A Waters Alliance HPLC system equipped with a 2996 PDA detector and a Superdex 75 10/300 GL column (GE Healthcare) was used to analyse the recombinant scFimH proteins. Each protein sample was injected into an HPLC column previously equilibrated in TBS and run at a solvent flow rate of $0.8 \mathrm{ml} \mathrm{min}^{-1}$ for $25 \mathrm{~min}$ at $20{ }^{\circ} \mathrm{C}$. For molecular mass determination the column was calibrated with the protein markers BSA (66 kDa), HRP (40 kDa) and RNase B $(18 \mathrm{kDa})$ (Sigma). The eluted proteins were detected by UV absorbance at $280 \mathrm{~nm}$.

Circular dichroism (CD) spectroscopy. The secondary structures of proteins were analysed by CD measurements in a JASCO J-715 spectropolarimeter (JASCO International). The proteins were dissolved in PBS (137 mM NaCl, $2.7 \mathrm{mM} \mathrm{KCl}, 10 \mathrm{mM} \mathrm{NaH}_{2} \mathrm{PO}_{4}, 2 \mathrm{mM}$ $\left.\mathrm{K}_{2} \mathrm{HPO}_{4}, \mathrm{pH} 7.4\right)$ and $\mathrm{CD}$ spectra were measured at $25{ }^{\circ} \mathrm{C}$ using a thermostat-controlled $0.1 \mathrm{~cm}$ cell. Spectra were recorded with a response time of $1.0 \mathrm{~s}$ and with $1.0 \mathrm{~nm}$ resolution. Each spectrum is the result of five spectra accumulated and averaged.

Real-time interaction analysis by surface plasmon resonance (SPR). The immobilization of high-mannose glycoproteins to CM5 (GE Healthcare) sensor chips has been described previously (Kisiela et al., 2005a), and the binding of the scFimH analytes was analysed using a BIAcore 1000 SPR instrument. All binding experiments were carried out at $25{ }^{\circ} \mathrm{C}$ with a $5 \mu \mathrm{l} \mathrm{min}{ }^{-1}$ flow rate. To determine FimH affinity to the immobilized proteins, $40 \mu \mathrm{l}$ of six different concentrations $(1 \mu \mathrm{M}, 500 \mathrm{nM}, 250 \mathrm{nM}, 125 \mathrm{~nm}, 62.5 \mathrm{nM}$ and $31.25 \mathrm{nM}$ ) of scFimH adhesive variant solutions, as well as sample buffer blank, were passed over the ligand-immobilized chip surface (association phase), followed by $8 \mathrm{~min}$ of dissociation with the running buffer. The same samples were passed over a control chip surface without immobilized ligand. Three replicates of each analyte concentration were injected. The resulting sensorgrams were obtained first by subtracting the buffer blank from curves recorded for the interactions of FimH proteins with these glycoproteins. Then the curves recorded when adhesins were passed over the blank surface were subtracted from such sensorgrams. The equilibrium constants were determined using BIAevaluation 3.1 software. For global fitting a 1:1 Langmuir binding model with an included mass transport step was applied based on criteria provided by the BIAevaluation handbook.

\section{RESULTS}

\section{Construction of scFimH variants of S. Enteritidis and $S$. Typhimurium}

To fill the hydrophobic groove exposed in the fimbrial domain of FimH proteins in the absence of FimF protein (Jones et al., 1995; Hancox et al., 1997; Choudhury et al., 1999), the strategy described by Barnhart et al. (2000) was used. The N-terminal extension of FimF, preceded by a short linker, was linked to the C-terminal end of FimH to allow the donor strand to supplement the hydrophobic groove in the fimbrial domain with the missing C-terminal
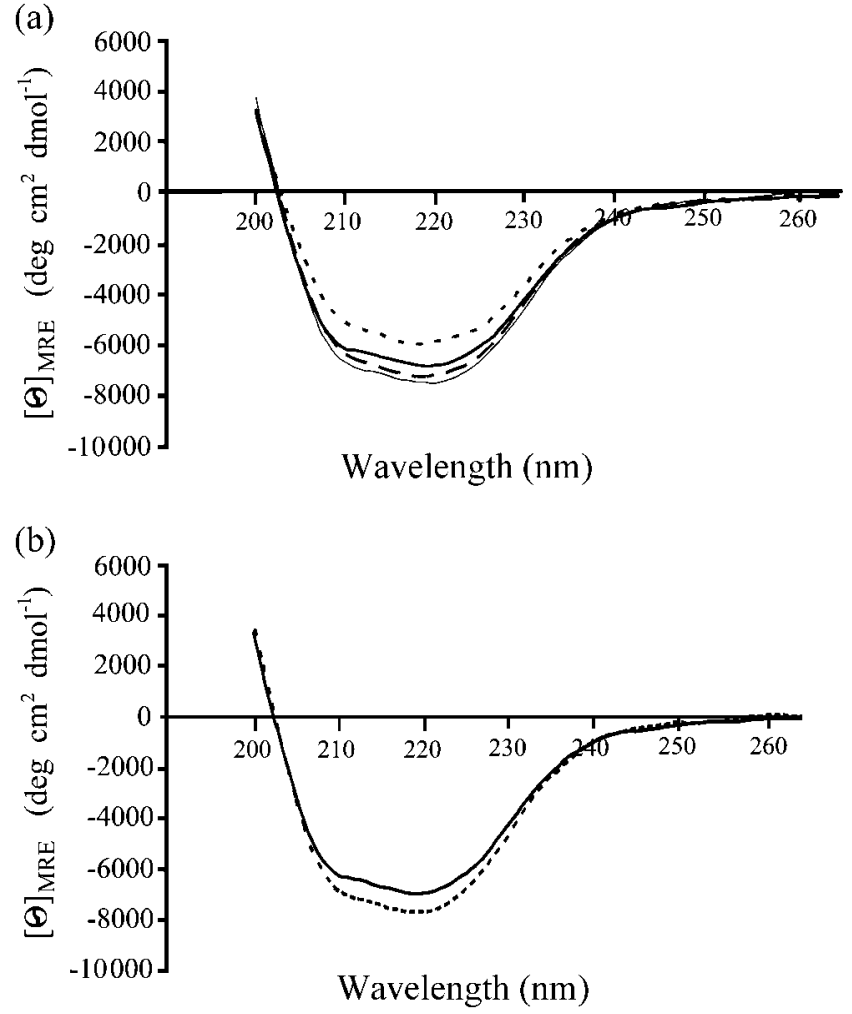

Fig. 2. (a) Far-UV CD spectra of scFimH adhesins with an $N-$ terminal extension of FimF $\left(0.1 \mathrm{mg} \mathrm{ml}^{-1}\right)$; short-dashed line, lowadhesive variant of $S$. Enteritidis; heavy solid line, high-adhesive variant of $S$. Enteritidis; long-dashed line, low-adhesive variant of $S$. Typhimurium; light solid line, high-adhesive variant of $S$. Typhimurium. (b) Far-UV CD spectra of the low-adhesive variant of the scFimH adhesin of $S$. Enteritidis with an $\mathrm{N}$-terminal extension from FimF $\left(0.1 \mathrm{mg} \mathrm{m}{ }^{-1}\right.$ ) purified in (solid line) denaturing (presence of $8 \mathrm{M}$ urea) and (dashed line) nondenaturing (without urea) conditions. Spectra were recorded with a response time of $1.0 \mathrm{~s}$ and a data point resolution of $1 \mathrm{~nm}$, using a cuvette with $0.1 \mathrm{~cm}$ path length. $[\theta]_{\text {MRE }}$ indicates mean residue ellipticity (in degrees $\mathrm{cm}^{2} \mathrm{dmol}^{-1}$ ). Five scans were averaged to obtain final spectra.

extension (Fig. 1a). These fusion proteins were named selfcomplemented FimHs (scFimHs).

The low- and high-adhesive variants of the $S$. Enteritidis and $S$. Typhimurium fimH genes extended by the $\mathrm{N}$-terminal extension of the fimF gene from $S$. Enteritidis were constructed as described in Methods and cloned into plasmid pET-22b containing a His affinity tag. Then, E. coli BL21 cells were transformed with the respective plasmids and the resulting recombinant proteins EscFimH/L (low-adhesive variant) and EscFimH/H (high-adhesive variant) of $S$. Enteritidis and $\mathrm{TscFimH} / \mathrm{L}$ (low-adhesive variant) and TscFimH/H (high-adhesive variant) of $S$. Typhimurium were purified by chromatography on an Ni-NTA affinity matrix. The identity of the scFimH proteins was confirmed first by SDS-PAGE 
(a)

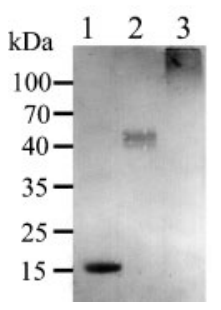

(b)

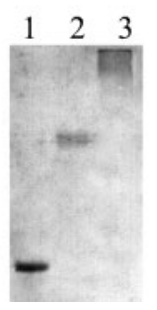

(c)

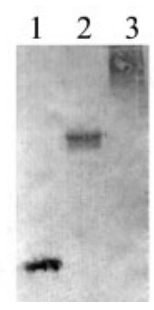

(d)

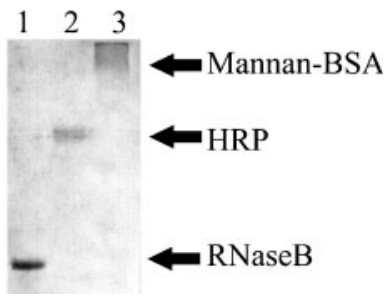

Fig. 3. Far-Western blot analysis of scFimH adhesins with an $\mathrm{N}$-terminal extension from the FimF protein binding to glycoproteins carrying high-mannose-type oligosaccharide chains. Binding of the (a) low- and (b) high-adhesive variants of scFimH from $S$. Enteritidis and (c) low- and (d) high-adhesive variants of scFimH from S. Typhimurium to RNase B (lane 1), HRP (lane 2) and mannan-BSA (lane 3). Each glycoprotein $(4 \mu \mathrm{g})$ was subjected to SDSPAGE under reducing conditions in a $10 \% \mathrm{gel}$ and transferred to nitrocellulose. The migration positions of protein standards (in $\mathrm{kDa}$ ) are indicated on the left. and then by Western blotting with rabbit polyclonal antiFimH antibodies and anti-myc mAb 9E10 (Fig. 1b).

\section{Molecular characterization of scFimH proteins}

To confirm that the EscFimH/L, EscFimH/H, TscFimH/L and $\mathrm{TscFimH} / \mathrm{H}$ proteins did not form aggregates, they were subjected to analytical HPLC using a Superdex 75 10/ 300 GL column. As expected, for each of the analysed proteins over $95 \%$ of the applied sample was present in monomeric form (Supplementary Fig. S1). The structural organization of the scFimH proteins was analysed using $\mathrm{CD}$. It was found that all four analysed adhesins (EscFimH/ L, EscFimH/H, TscFimH/L and TscFimH/H) possessed
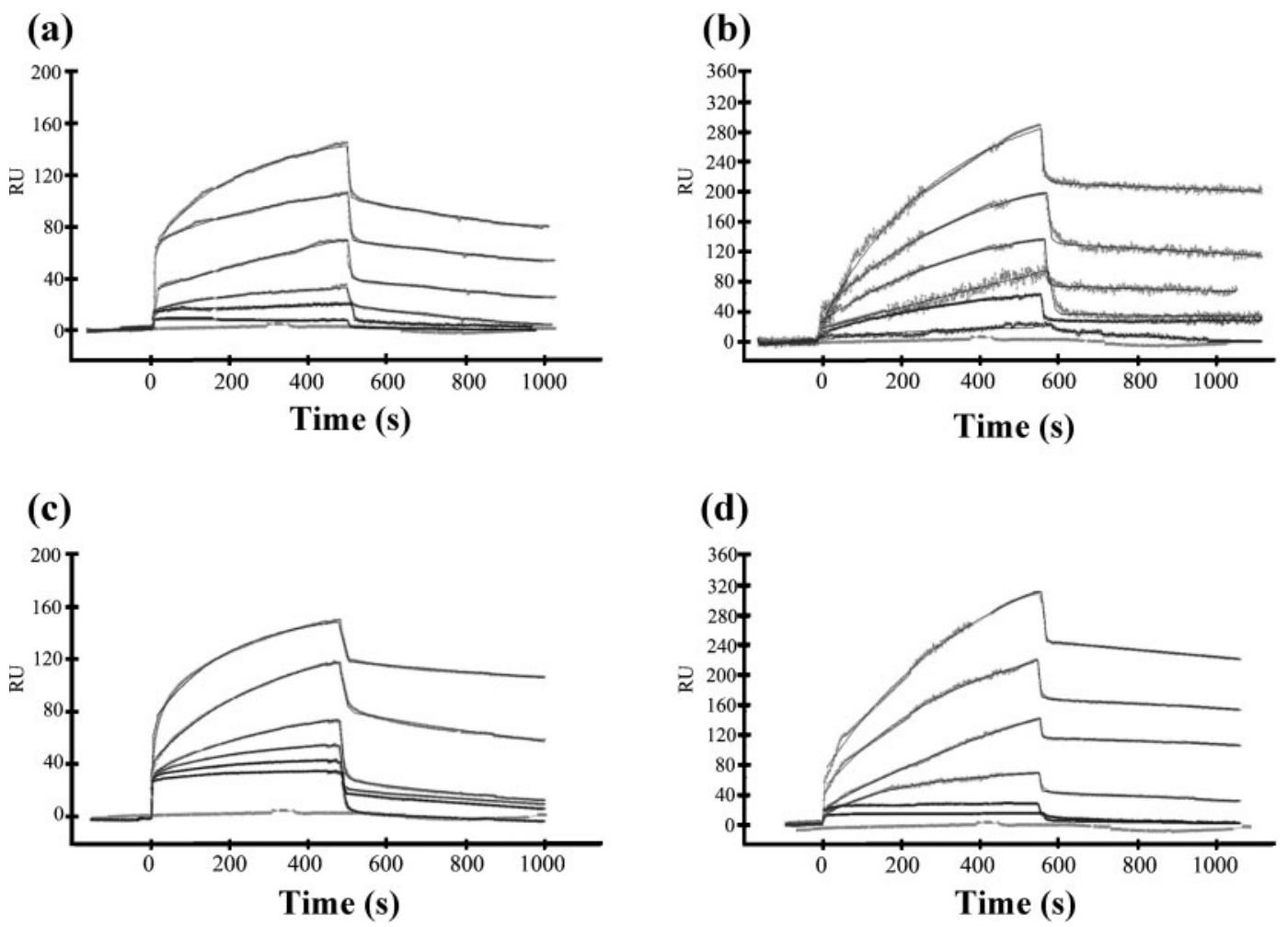

Fig. 4. Interaction of scFimH proteins of $S$. Enteritidis and $S$. Typhimurium possessing an $\mathrm{N}$-terminal extension from the FimF protein with immobilized RNase B analysed with an SPR biosensor. (a) Low-adhesive variant of S. Enteritidis; (b) high-adhesive variant of S. Enteritidis; (c) low-adhesive variant of $S$. Typhimurium; (d) high-adhesive variant of $S$. Typhimurium. Grey lines represent different concentrations of analyte $(1 \mu \mathrm{M}, 500 \mathrm{nM}, 250 \mathrm{nM}, 125 \mathrm{nM}, 62 \mathrm{nM}$ and $31 \mathrm{nM})$ in TBS. Binding data were collected at a flow rate of $5 \mu \mathrm{min}^{-1}$. Black solid lines represent the best global fit of the experimental data to a $1: 1$ Langmuir reaction model with an included mass transport step (BIAevaluation 3.1 software). RU, relative units. 
(a)

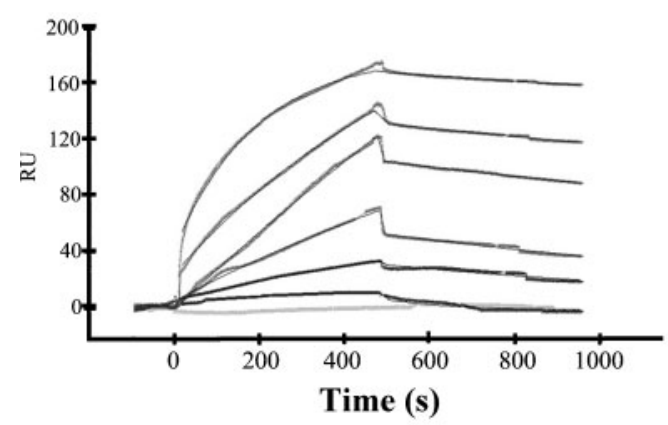

(c)

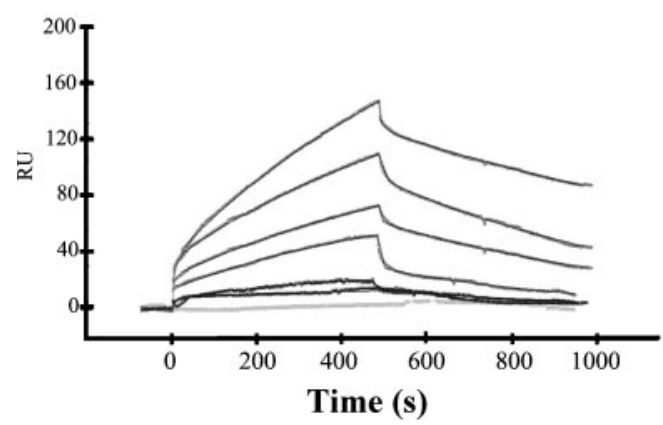

(b)

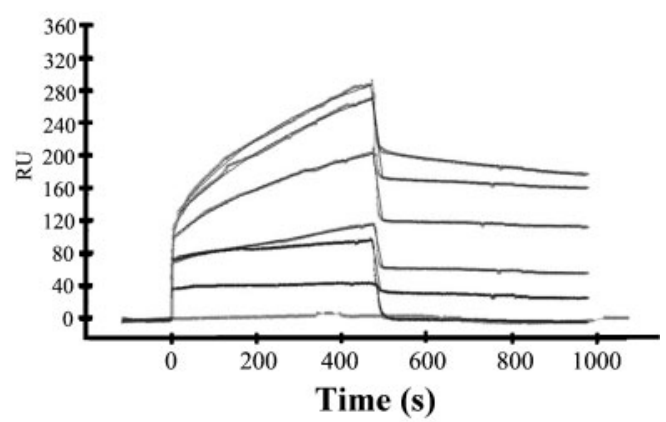

(d)

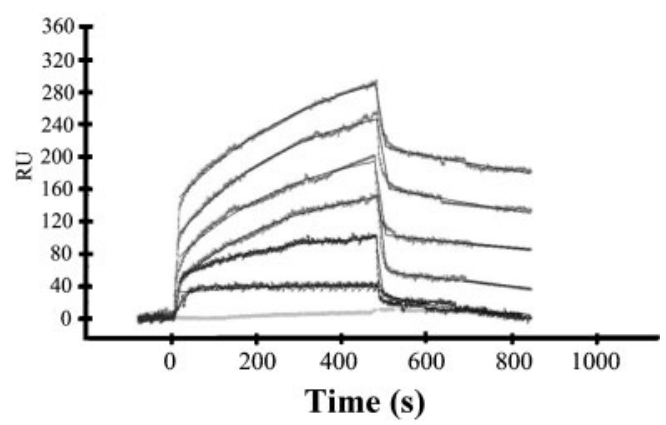

Fig. 5. Interaction of scFimH proteins of $S$. Enteritidis and $S$. Typhimurium possessing an $\mathrm{N}$-terminal extension from the FimF protein with immobilized HRP analysed with an SPR biosensor. (a) Low-adhesive variant of S. Enteritidis; (b) high-adhesive variant of S. Enteritidis; (c) low-adhesive variant of $S$. Typhimurium; (d) high-adhesive variant of S. Typhimurium. Grey lines represent different concentrations of analyte $(1 \mu \mathrm{M}, 500 \mathrm{nM}, 250 \mathrm{nM}, 125 \mathrm{nM}, 62 \mathrm{nM}$ and $31 \mathrm{nM})$ in TBS. Binding data were collected at a flow rate of $5 \mu \mathrm{min}^{-1}$. Black solid lines represent the best global fit of the experimental data to a $1: 1$ Langmuir reaction model with an included mass transport step (BIAevaluation 3.1 software). RU, relative units.

essentially identical, extensive secondary structure (Fig. 2a). No differences in CD spectra were found between scFimH proteins purified in denaturing (presence of $8 \mathrm{M}$ urea) conditions and those purified in non-denaturing (without urea) conditions (Fig. 2b).

\section{Binding properties of G61A and F118S double mutants of scFimHs}

The ability of the low- and high-adhesive variants of scFimH proteins to bind mannose-rich carbohydrate structures was studied by Western blotting with RNase B, HRP and the synthetic glycoconjugate mannan-BSA (Fig. 3). The binding was completely inhibited by preincubation with $0.2 \mathrm{M}$ D-mannose (data not shown). There was no difference in binding between the low- and high-adhesive variants of the FimH adhesins from $S$. Enteritidis and $S$. Typhimurium.

The binding of $S$. Enteritidis and $S$. Typhimurium low- and high-adhesive variants of the scFimH adhesins to RNase B (Fig. 4), HRP (Fig. 5) and mannan-BSA (Fig. 6) was analysed by SPR. The sensorgrams were obtained first by subtracting the buffer blank from curves recorded for the interactions of FimH proteins with these glycoproteins. Then the curves, recorded when adhesins were passed over the blank surface, were subtracted from such sensorgrams. Association $\left(\mathrm{k}_{\mathrm{a}}\right)$ and dissociation $\left(\mathrm{k}_{\mathrm{d}}\right)$ rate constants were calculated using a 1:1 Langmuir model with an included mass transport step, based on the criteria provided by the BIAevaluation handbook. The SPR study revealed profound differences in $K_{D}$ values, estimated as $k_{d} / k_{a}$ ratio, between the high- and low-adhesive variants of the scFimH proteins from both Salmonella serovars studied (Table 1).

\section{Binding properties of Gly61Ala or Phe118Ser single mutants of $\mathrm{S}$. Enteritidis scFimH}

To address the question of which amino acid substitution (Gly61Ala or Phe118Ser) was mainly responsible for the differences in binding affinity of the low- and highadhesive variants of the FimH adhesins, fimH gene mutants of the low-adhesive variant of $S$. Enteritidis with single Gly61Ala or Phe118Ser mutations were constructed as described in Methods. The resulting plasmids were used to transform E. coli BL21 cells, and the recombinant scFimH 

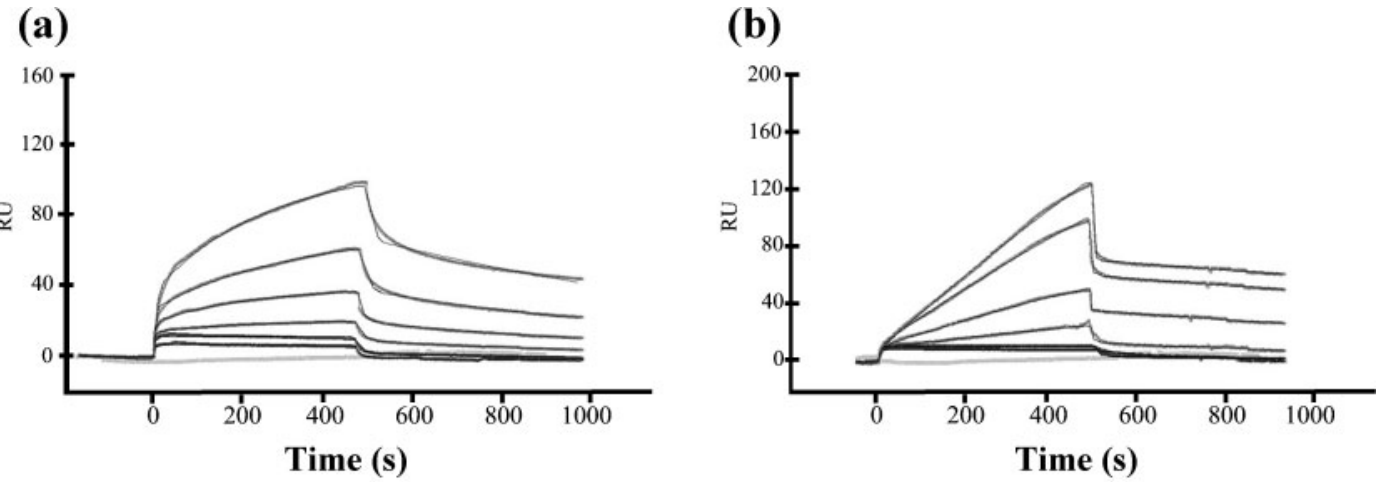

(c)
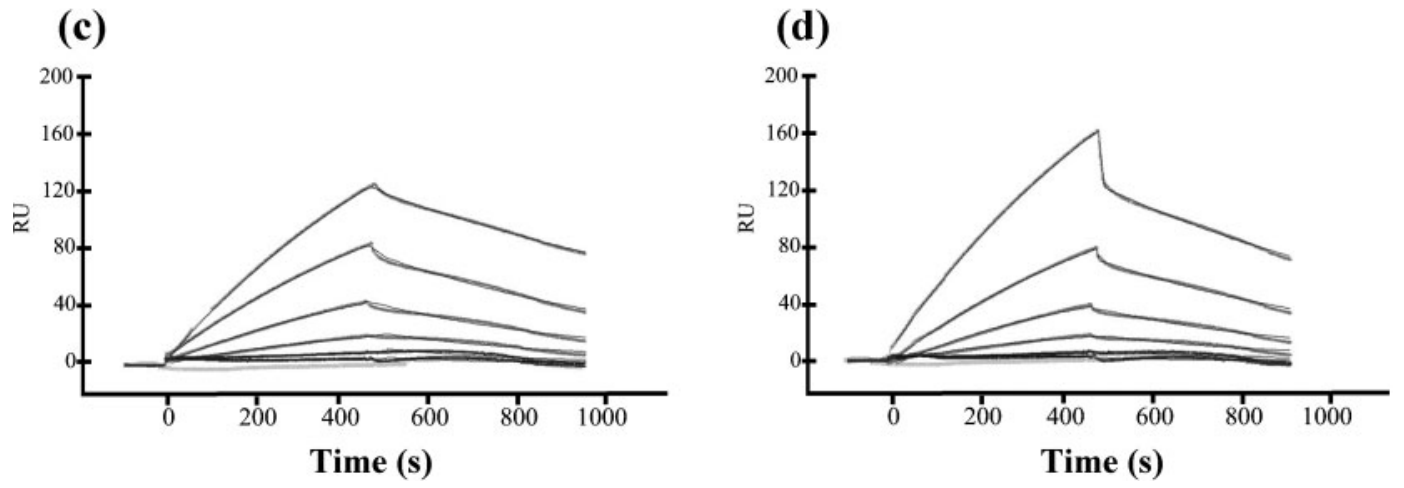

Fig. 6. Interaction of scFimH proteins of $S$. Enteritidis and $S$. Typhimurium possessing an $\mathrm{N}$-terminal extension from the FimF protein with immobilized mannan-BSA analysed with an SPR biosensor. (a) Low-adhesive variant of S. Enteritidis; (b) highadhesive variant of $S$. Enteritidis; (c) low-adhesive variant of $S$. Typhimurium; (d) high-adhesive variant of $S$. Typhimurium. Grey lines represent different concentrations of analyte $(1 \mu \mathrm{M}, 500 \mathrm{nM}, 250 \mathrm{nM}, 125 \mathrm{nM}, 62 \mathrm{nM}$ and $31 \mathrm{nM})$ in TBS. Binding data were collected at a flow rate of $5 \mu \mathrm{min}^{-1}$. Black solid lines represent the best global fit of the experimental data to a $1: 1$ Langmuir reaction model with an included mass transport step (BIAevaluation 3.1 software). RU, relative units.

adhesins, named EscFimH/L(Gly61Ala) and EscFimH/ L(Phe118Ser), after chromatography on an Ni-NTA affinity matrix, were analysed by SDS-PAGE and Western blotting. The same pattern of Coomassie blue staining and immunostaining with anti-FimH antibodies as the lowand high-adhesive variants of other scFimH adhesins was observed (Supplementary Fig. S2a).

Both mutants of the $S$. Enteritidis scFimH low-adhesive variant carrying the single mutations Gly61Ala or Phe118Ser bound to glycoproteins carrying high-mannose glycoproteins to similar extents, as shown by far-Western blotting (Supplementary Fig. S2b). However, striking differences in the association $\left(\mathrm{k}_{\mathrm{a}}\right)$ and dissociation $\left(\mathrm{k}_{\mathrm{d}}\right)$ rate constants were found between the EscFimH/ L(Gly61Ala) and EscFimH/L(Phe118Ser) adhesins when their interactions with RNase B, HRP and mannose-BSA were studied by SPR (Fig. 7). The mutant identical to the low-adhesive variant of the FimH protein from $S$. Enteritidis except at position 118, where phenylalanine was replaced by serine, bound to all three analysed highmannose glycoproteins with much higher affinity than the parental scFimH protein (Table 1). Interestingly, the second mutant, identical to the low-adhesive variant except at position 61 , where glycine was exchanged by alanine, bound to the same glycoproteins with $K_{D}$ values similar to those of the non-mutated protein (Table 1).

\section{DISCUSSION}

Our previous study of FimH sequences from different avian and human isolates of $S$. Enteritidis revealed that all the analysed isolates express the same allelic variant of FimH (Kisiela et al., 2005b), which was first found in the $S$. Typhimurium SL1344 strain by Boddicker et al. (2002). This strain of $S$. Typhimurium bound weakly to HEp-2 cells, in contrast to the high-adhesive strain LB5010, which attached strongly to those cells. The authors claimed that the different properties of the low- and high-adhesive variants of FimH proteins depend mostly on two amino acid residues located at positions 61 and 118. A glycine residue at position 61 and phenylalanine at position 118 were found in the low-adhesive variant of the FimH adhesin, and the high-adhesive variant of this protein had alanine at position 61 and serine at position 118. Therefore, 
Table 1. Dissociation constants of scFimH adhesins determined by SPR analysis

\begin{tabular}{|llc|}
\hline FimH variant & \multicolumn{1}{c|}{ Ligand } & $\mathbf{K}_{\mathbf{D}}(\mathbf{M})$ \\
\hline EscFimH/L & RNase B & $5.60 \times 10^{-8} \pm 1.06 \times 10^{-8}$ \\
& HRP & $2.30 \times 10^{-8} \pm 6.30 \times 10^{-9}$ \\
& Mannan-BSA & $3.23 \times 10^{-6} \pm 9.10 \times 10^{-7}$ \\
EscFimH/H & RNase B & $6.33 \times 10^{-9} \pm 1.25 \times 10^{-9}$ \\
& HRP & $2.60 \times 10^{-9} \pm 7.80 \times 10^{-10}$ \\
& Mannan-BSA & $2.00 \times 10^{-7} \pm 3.30 \times 10^{-8}$ \\
TscFimH/L & RNase B & $3.48 \times 10^{-8} \pm 7.92 \times 10^{-9}$ \\
& HRP & $4.94 \times 10^{-8} \pm 5.23 \times 10^{-9}$ \\
& Mannan-BSA & $9.00 \times 10^{-6} \pm 6.03 \times 10^{-7}$ \\
TscFimH/H & RNase B & $8.70 \times 10^{-9} \pm 1.10 \times 10^{-9}$ \\
& HRP & $8.36 \times 10^{-9} \pm 1.07 \times 10^{-9}$ \\
EscFimH/L(Gly61Ala) & Mannan-BSA & $9.50 \times 10^{-7} \pm 2.11 \times 10^{-8}$ \\
& RNase B & $3.91 \times 10^{-8} \pm 3.68 \times 10^{-9}$ \\
EscFimH/L(Phe118Ser) & HRP & $2.50 \times 10^{-8} \pm 6.59 \times 10^{-9}$ \\
& Mannan-BSA & $3.50 \times 10^{-6} \pm 8.82 \times 10^{-7}$ \\
& RNase B & $5.67 \times 10^{-9} \pm 1.24 \times 10^{-9}$ \\
& HRP & $7.40 \times 10^{-9} \pm 1.33 \times 10^{-9}$ \\
& Mannan-BSA & $2.44 \times 10^{-7} \pm 7.70 \times 10^{-8}$ \\
\hline
\end{tabular}

we asked whether the same amino acid substitutions (Gly61Ala and Phe118Ser) could be responsible for similar changes in the binding properties of the low-adhesive FimH from $S$. Enteritidis. In contrast to the earlier study, the binding properties of the FimH adhesins were studied at the molecular level using purified recombinant wild-type and mutated proteins. As the purified native FimH proteins have a high tendency to form aggregates, which is a major problem when they are analysed by SPR (Van der Merwe \& Barclay, 1996), we constructed fusion FimH proteins containing an additional amino acid sequence representing an N-terminal extension of the FimF protein. Such a modification, by taking advantage of a donor strand exchange mechanism, enabled the completion of the immunoglobulin fold in the FimH protein, closing the hydrophobic groove in the fimbrial domain (Jones et al., 1995; Barnhart et al., 2000). As expected, self-complemented adhesins ( $\mathrm{scFimH}$ ) did not form aggregates and were more stable (resistant to proteolytic cleavage) than native FimH.

As shown by SPR, the low-adhesive FimH variant of $S$. Enteritidis bound with a much higher $\mathrm{K}_{\mathrm{D}}$ (about 10 times) to RNase B, HRP and mannan-BSA than its high-adhesive counterpart. Less profound differences in $K_{D}$ values were found between the low- and high-adhesive variants from $S$. Typhimurium. The interactions of the low-adhesive variants with RNase B and HRP gave dissociation constants about five to six times higher than the high-adhesive proteins. The distinctly higher $\mathrm{K}_{\mathrm{D}}$ (about 10 times) was found only for mannan-BSA. The $\mathrm{K}_{\mathrm{D}}$ values obtained in this study for the interactions of the low-adhesive variants with all three high-mannose glycoproteins are much lower than those previously published (Kisiela et al., 2006). These discrepancies are most probably the result of the different approach applied in the present study. Instead of native FimH protein, with a high tendency to form aggregates, fully soluble chimeric adhesin with an N-terminal extension from FimF was used. In addition, this SPR study was performed in the absence of $0.2 \mathrm{M}$ guanidine hydrochloride. Taken together, our study revealed on the level of isolated FimH proteins the existence of low- and highadhesive variants of the FimH adhesins not only for $S$. Typhimurium but also for $S$. Enteritidis, although their binding characteristics to high-mannose structures were slightly different. Therefore, these data suggest that, in addition to amino acid residues at positions 61 and 118, other amino acid substitutions could influence the specificity of FimH variants for different ligands. The low- and high-adhesive variants of $S$. Enteritidis and $S$. Typhimurium differ at four amino acid positions (R126L, S131Y, M137K and N317I). The same number of differences in amino acid sequences was also found between high-adhesive variants of these two Salmonella serovars (R126L, S131Y, M137K and N317I). As the lowand high-adhesive allelic variants of FimH adhesin from $S$. Enteritidis and $S$. Typhimurium extracted from fimbrial shafts bound with distinctly different affinities to glycoproteins carrying high-mannose structures, our data strongly suggest that variations in binding specificity are dependent only on the structure of the FimH protein itself, and not on the fimbrial shaft, as has been proposed by several authors (Thankavel et al., 1999; Duncan et al., 2005; Madison et al., 1994). Based on the results obtained with the FimH proteins of E. coli and Klebsiella pneumoniae, which show that the affinity of the adhesin for mannosylated ligands can be directly regulated by the pilin domain by the use of the allosteric catch bond mechanism (Aprikian et al., 2007; Stahlhut et al., 2009), we speculate that a similar mechanism could be responsible for the differences in $K_{D}$ values obtained for high- and lowadhesive variants of FimH adhesins from Salmonella. 


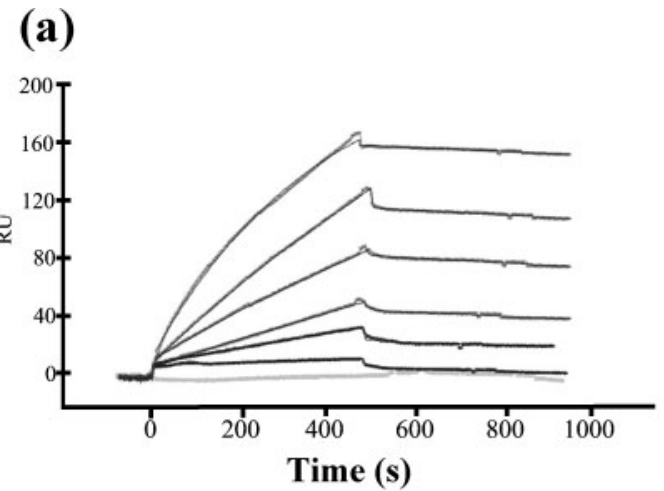

(c)

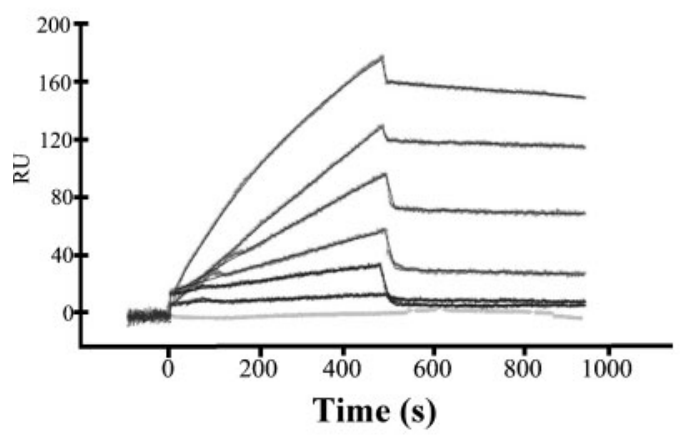

(e)

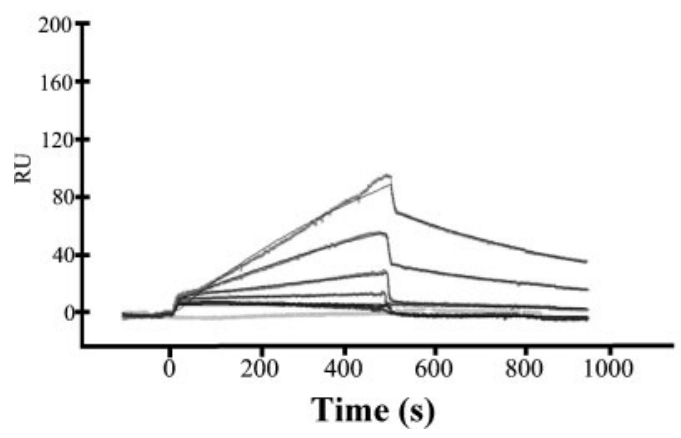

(b)

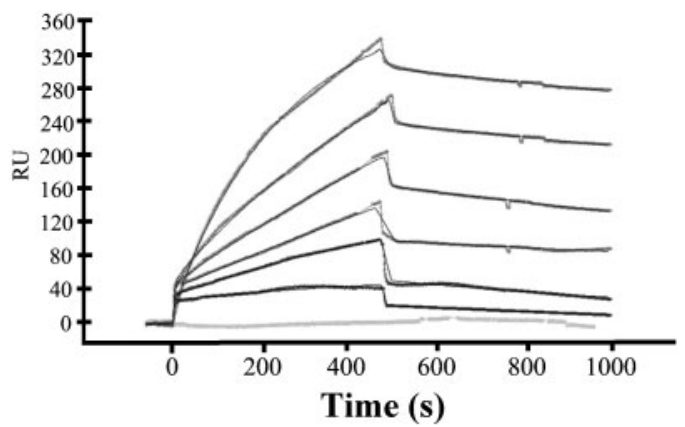

(d)

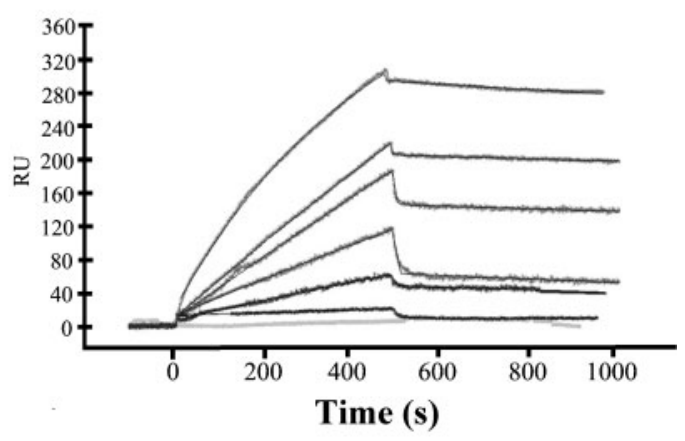

(f)

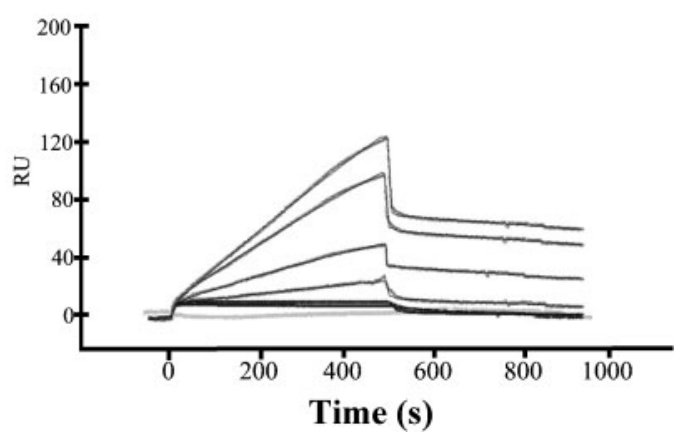

Fig. 7. Interaction of (a, c, e) EscFimH/L(Gly61Ala) and (b, d, f) EscFimH/L(Phe118Ser) mutants with immobilized RNase B, HRP and mannan-BSA analysed with an SPR biosensor. The EscFimH(Gly61Ala) mutant, constructed by site-directed mutagnesis, was identical to the low-adhesive variant of the scFimH adhesin of $S$. Enteritidis except at the position corresponding to amino acid position 61, where glycine was replaced by alanine (Gly61Ala); the EscFimH(Phe118Ser) mutant was identical to the low-adhesive variant of the scFimH adhesin of $S$. Enteritidis except at the position corresponding to amino acid 118, where phenylalanine was replaced by serine (Phe118Ser). Grey lines represent different concentrations of analyte $(1 \mu \mathrm{M}, 500 \mathrm{nM}, 250 \mathrm{nM}, 125 \mathrm{nM}, 62 \mathrm{nM}$ and $31 \mathrm{nM})$ in TBS. Binding data were collected at a flow rate of $5 \mu \mathrm{min}{ }^{-1}$. Black solid lines represent the best global fit of the experimental data to a $1: 1$ Langmuir reaction model with an included mass transport step (BIAevaluation 3.1 software). RU, relative units.

Our data on the involvement of amino acid residues 61 and 118 in determining the binding properties of low- and high-adhesive variants of FimH adhesin differ from those of Boddicker et al. (2002), who found that Ala61 is mostly responsible for supporting the attachment of $S$. Typhimurium LB5010 to HEp-2 cells. Mutated low- adhesive FimH protein from $S$. Enteritidis, in which the glycine at position 61 was replaced by the alanine found in the high-adhesive variant of $S$. Typhimurium, bound with almost the same association $\left(\mathrm{k}_{\mathrm{a}}\right)$ and dissociation $\left(\mathrm{k}_{\mathrm{d}}\right)$ rate constants to all analysed glycoproteins as the native adhesin. In contrast, when phenylalanine at position 118 
was replaced by the serine present in the high-adhesive variant of FimH, the mutated form of the low-adhesive protein from $S$. Enteritidis interacted with higher affinity with mannan-BSA ( $K_{D}$ about 10 times lower) as well as with RNase $B$ and HRP ( $K_{D}$ about five to seven times lower). Therefore, using mutated forms of low-adhesive FimH from S. Enteritidis (Gly61Ala and Phe118Ser), our SPR study points to Ser118 as the major determinant of the high-adhesive phenotype of type 1 fimbriae from $S$. Enteritidis.

In summary, we have obtained for the first time functional full-size FimH adhesins as stable and highly soluble proteins which can be used to study structure-function relationships with their respective ligands at the molecular level. SPR analysis with highly purified fusion FimH proteins revealed the existence of high- and low-adhesive allelic variants not only in $S$. Typhimurium but also in $S$. Enteritidis. Our studies are believed to be so far the only ones in which the functional differences observed with whole fimbriated bacteria can be reproduced at the level of purified adhesin. They strongly suggest that the adhesive properties of type 1 fimbriae are determined only by structural differences in the FimH proteins and are not influenced by the fimbrial shaft on which the adhesin is located. Site-directed mutagenesis of the low-adhesive variant from $S$. Enteritidis also indicated the critical role of phenylalanine versus serine substitution at position 118 in the binding properties of the FimH adhesin.

\section{ACKNOWLEDGEMENTS}

This work was supported by grant 2 P06K 02330 of the Ministry of Science and Higher Education (Poland). The authors are grateful to Professor Aleksander F. Sikorski and Dr Dagmara Kisiela for their critical reading of the manuscript and valuable discussions.

\section{REFERENCES}

Abraham, S. N., Sun, D., Dale, J. B. \& Beachey, E. H. (1988), Conservation of the D-mannose-adhesion protein among type 1 fimbriated members of the family Enterobacteriaceae. Nature 336, 682-684.

Allen-Vercoe, E. \& Woodward, M. J. (1999). The role of flagella, but not fimbriae, in the adherence of Salmonella enterica serotype Enteritidis to chick gut explants. J Med Microbiol 48, 771-780.

Allen-Vercoe, E., Sayers, A. R. \& Woodward, M. J. (1999). Virulence of Salmonella enterica serotype Enteritidis aflagellate and afimbriate mutants in a day-old chick model. Epidemiol Infect 122, 395-402.

Aprikian, P., Tchesnokova, V., Kidd, B., Yakovenko, O., YarovYarovoy, V., Trinchina, E., Vogel, V., Thomas, W. \& Sokurenko, E. (2007). Interdomain interaction in the FimH adhesin of Escherichia coli regulates the affinity to mannose. J Biol Chem 282, 23437-23446.

Barnhart, M. M., Pinkner, J. S., Soto, G. E., Sauer, F. G., Langermann, S. Waksman, G., Frieden, C. \& Hultgren, S. J. (2000). PapD-like chaperones provide the missing information for folding of pilin proteins. Proc Natl Acad Sci U S A 97, 7709-7714.

Boddicker, J. D., Ledeboer, N. A., Jagnow, J., Jones, B. D. \& Clegg, S. (2002). Differential binding to and biofilm formation on, HEp-2 cells by Salmonella enterica serovar Typhimurium is dependent upon allelic variation in the $\mathrm{fimH}$ gene of the fimH gene cluster. $\mathrm{Mol}$ Microbiol 45, 1255-1265.

Choudhury, D., Thompson, A., Stojanoff, V., Langermann, S., Pinkner, J., Hultgren, S. J. \& Knight, S. D. (1999). X-ray structure of the FimC-FimH chaperone-adhesin complex from uropathogenic Escherichia coli. Science 285, 1061-1066.

De Buck, J., Van Immerseel, F., Meulemans, G., Haesebrouck, F. \& Ducatelle, R. (2003). Adhesion of Salmonella enterica serotype Enteritidis isolates to chicken isthmal glandular secretions. Vet Microbiol 93, 223-233.

Dibb-Fuller, M. P. \& Woodward, M. J. (2000). Contribution of fimbriae and flagella of Salmonella enteritidis to colonization and invasion of chicks. Avian Pathol 29, 295-304.

Dibb-Fuller, M. P., Allen-Vercoe, E., Thorns, C. J. \& Woodward, M. J. (1999). Fimbriae- and flagella-mediated association with and invasion of cultured epithelial cells by Salmonella enteritidis. Microbiology 145, 1023-1031.

Duguid, J. P., Anderson, E. S. \& Campbell, I. (1966). Fimbriae and adhesive properties in salmonellae. J Pathol Bacteriol 92, 107-138.

Duncan, M. J., Mann, E. L., Cohen, M. S., Ofek, I., Sharon, N. \& Abraham, S. N. (2005). The distinct binding specificities exhibited by enterobacterial type 1 fimbriae are determined by their fimbrial shafts. J Biol Chem 280, 37707-37716.

Ewen, S. W., Naughton, P. J., Grant, G., Sojka, M., Allen-Vercoe, E., Bardocz, S., Thorns, C. J. \& Pusztai, A. (1997). Salmonella enterica var Typhimurium and Salmonella enterica var Enteritidis express type 1 fimbriae in the rat in vivo. FEMS Immunol Med Microbiol 18, 185192.

Firon, N., Ofek, I. \& Sharon, N. (1983). Carbohydrate specificity of the surface lectins of Escherichia coli, Klebsiella pneumoniae and Salmonella typhimurium. Carbohydr Res 120, 235-249.

Firon, N., Ofek, I. \& Sharon, N. (1984). Carbohydrate-binding sites of the mannose-specific fimbrial lectins of Enterobacteria. Infect Immun 43, 1088-1090.

Guo, A., Lasaro, M. A., Sirard, J.-C., Kraehenbuhl, P. \& Schifferli, D. M. (2007). Adhesin-dependent binding and uptake of Salmonella enterica serovar Typhimurium by dendritic cells. Microbiology 153, 1059-1069.

Hancox, L. S., Yeh, K. S. \& Clegg, S. (1997). Construction and characterization of type 1 non-fimbriate and non-adhesive mutants of Salmonella typhimurium. FEMS Immunol Med Microbiol 19, 289296.

Jones, C. H., Pinkner, J. S., Roth, R., Heuser, J., Nicholes, A. V., Abraham, S. N. \& Hultgren, S. J. (1995). FimH adhesin of type 1 pili is assembled into a fibrillar tip structure in the Enterobacteriaceae. Proc Natl Acad Sci U S A 92, 2081-2085.

Kanska, U., Budzynska, R., Nevozhay, D. \& Boratynski, J. (2008). Preparation of mannan-protein conjugates using high-temperature glycation. Biotechnol Appl Biochem 49, 57-64.

Kisiela, D., Kiczak, L., Kuźmińska, M., Kuczkowski, M., Franiczek, R. \& Ugorski, M. (2005a). Analysis of the fimH gene coding type 1 fimbriae adhesin of Salmonella enterica serovar Enteritidis. Med Welt 61, 1259-1262.

Kisiela, D., Sapeta, A. M., Kuczkowski, M., Stefaniak, T., Wieliczko, A. \& Ugorski, M. (2005b). Characterization of FimH adhesins expressed by Salmonella enterica serovars Gallinarum biovars Gallinarum and Pullorum: reconstitution of mannose-binding properties by single amino acid substitution. Infect Immun 73, 6187-6190.

Kisiela, D., Laskowska, A., Sapeta, A., Kuczkowski, M., Wieliczko, A. \& Ugorski, M. (2006). Functional characterization of the FimH 
adhesin from Salmonella enterica serovar Enteritidis. Microbiology 152, 1337-1346.

Krogfelt, K. A., Bergmans, H. \& Klemm, P. (1990). Direct evidence that the FimH protein is the adhesin of Escherichia coli type 1 fimbriae. Infect Immun 58, 1995-1999.

Madison, B., Ofek, I., Clegg, S. \& Abraham, S. N. (1994). Type 1 fimbrial shafts of Escherichia coli and Klebsiella pneumoniae influence sugar-binding specificities of their FimH adhesins. Infect Immun 62, 843-848.

Naughton, P. J., Grant, G., Bardocz, S., Allen-Vercoe, E., Woodward, M. J. \& Pusztai, A. (2001). Expression of type 1 fimbriae (SEF 21) of Salmonella enterica serotype Enteritidis in the early colonization of the rat intestine. J Med Microbiol 50, 191-197.

Rajashekara, G., Munir, S., Alexeyev, M. F., Halvorson, D. A., Wells, C. L. \& Nagaraja, K. V. (2000). Pathogenic role of SEF14, SEF17, and SEF21 fimbriae in Salmonella enterica serovar Enteritidis infection of chickens. Appl Environ Microbiol 66, 1759-1763.

Sokurenko, E. V., Courtney, H. S., Ohman, D. E., Klemm, P. \& Hasty, D. L. (1994). FimH family of type 1 fimbrial adhesins: functional heterogeneity due to minor sequence variations among fim $H$ genes. J Bacteriol 176, 748-755.
Sokurenko, E. V., Courtney, H. S., Maslow, J., Siitonen, A. \& Hasty, D. L. (1995). Quantitative differences in adhesiveness of type 1 fimbriated Escherichia coli due to structural differences in $\mathrm{fimH}$ genes. J Bacteriol 177, 3680-3686.

Stahlhut, S. G., Tchesnokova, V., Struve, C., Scott, J. W., Chattopadhyay, S., Yakovenko, O., Aprikian, P., Sokurenko, E. V. \& Krogfelt, K. A. (2009). Comparative structure-function analysis of mannose-specific FimH adhesins from Klebsiella pneumoniae and Escherichia coli. J Bacteriol 191, 6592-6601.

Thankavel, K., Shah, A. H., Cohen, M. S., Ikeda, T., Lorenz, R. G., Curtiss, R. \& Abraham, S. N. (1999). Molecular basis for the enterocyte tropism exhibited by Salmonella typhimurium type 1 fimbriae. J Biol Chem 274, 5797-5809.

Thorns, C. J. (1995). Salmonella fimbriae: novel antigens in the detection and control of Salmonella infections. Br Vet J 151, 643658.

Van der Merwe, P. A. \& Barclay, A. N. (1996). Analysis of celladhesion molecule interactions using surface plasmon resonance. Curr Opin Immunol 8, 257-261.

Edited by: J. G. Shaw 